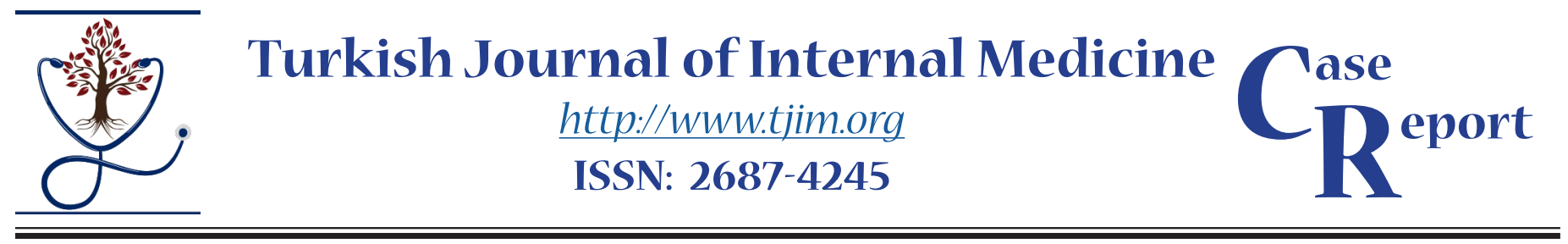

\title{
Acute Aortic Dissection Case with Stroke Presentation
}

\author{
Meral Seferoglu $^{1}$ (D), Nizameddin Koca ${ }^{2}$ (D), Baris Sensoy ${ }^{3}$ (D) \\ ${ }^{1}$ Department of Neurology, University of Health Sciences, Bursa Yüksek İhtisas Training and Research Hospital, Bursa, Turkey \\ ${ }^{2}$ Department of Internal Medicine, University of Health Sciences, Bursa Yüksek İhtisas Training and Research Hospital, Bursa, \\ Turkey \\ ${ }^{3}$ Department of Cardiology, University of Health Sciences, Bursa Yüksek İhtisas Training and Research Hospital, Bursa, Turkey
}

\begin{abstract}
The correct diagnosis of acute aortic syndrome complicated by neurologic symptoms is essential. Performing thrombolytic therapy for acute-stage cerebral infarction would probably be the reason for fatal outcomes. We herein report the case of a 63-year-old man who presented with left hemiparesis and paraparesis. An acute myocardial infarction was excluded by electrocardiography and blood tests. Head computed tomography (CT) showed no remarkable findings. Although there was no chest pain, it was found in CT-angiography that he has an intramural hematoma in the ascending aorta and a severe dissection in the descending aorta. Aortic dissection may mimic acute stroke, in order to make the right decision about the treatment of acute aortic syndrome with neurologic complications, the benefits and risks should therefore be considered in individual patients.
\end{abstract}

Keywords: aort dissection, paraparesia, stroke, tissue plasminogen activator

\section{Introduction}

Acute aortic syndrome (AAS) is a life- and paraplegia, which could cause difficulty to threatening medical emergency associated diagnose early and treat in clinical settings. ${ }^{1}$ We with high rates of morbidity and mortality. The symptoms of AAS can be variable and may mimic those of more common conditions herein report a patient with an AAS presenting with signs of acute stroke as left hemiparesis and paraparesis of lower extremities. such as myocardial infarction, stroke, shock,

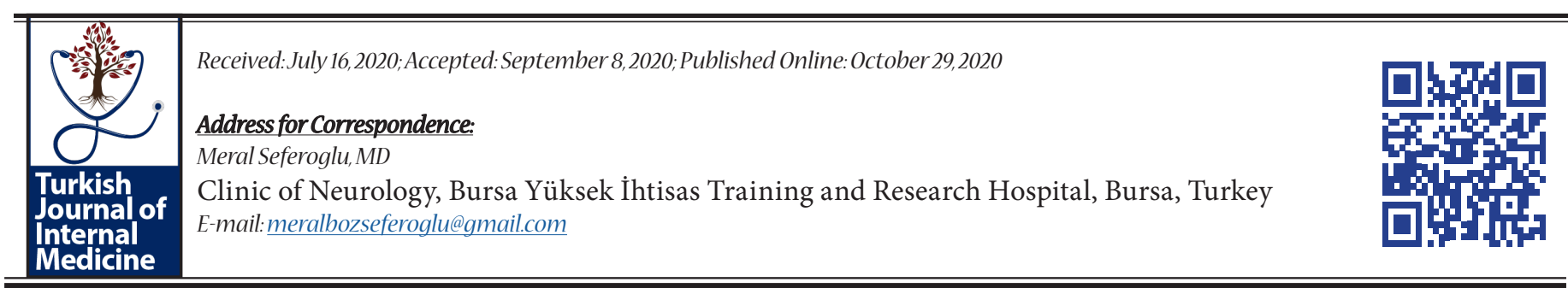




\section{Case Report}

A 63-year-old man admitted to emergency department with the complaints of acute onset of left upper extremity hemiparesis and asymmetric paraparesis with hypoesthesia for two hours. It was suspected that he may have an acute ischemic stroke and thrombolytic treatment was planned. So far he was a healthy man without any cardiovascular risk factors. Upon further questioning, the patient denied any kind of trauma, and skip a dose of medications. On physical exam, he was anxious and had a heart rate of 110 beats per minute. An acute myocardial infarction was excluded by electrocardiography and blood tests. A transthoracic echocardiography showed no definite abnormalities in the ascending aorta, valve, regional wall motion, and global left ventricular function in conventional echocardiographic views (data not shown). Head computed tomography (CT) and magnetic resonance imaging (MRI) showed no remarkable findings (Figure 1). In the clinical evaluation it was revealed that he has a pulse deficit in the leftsided extremities and $40 \mathrm{mmHg}$ blood pressure difference was observed between the upper extremities. Although there was no chest pain, CT angiography revealed an aortic dissection which extended to the descending aorta (Stanford type A, Figure 2). We therefore carried out the strict control of the patient's blood pressure and gave up the decision of thrombolytic treatment aside. He underwent emergency allograft replacement, but he died during the operation.

It is extremely difficult to diagnose painless acute aortic dissection (AAD) that presents with neurologic symptoms solely. It is important to exclude AAD in patients with acute neurological symptoms that needs to receive tissue plasminogen activator (tPA), due to their fatal course.

\section{Discussion}

AAS complicated by neurologic symptoms or neurologic complications is not rare. This clinical scenario may lead to inappropriate thrombolytic or anticoagulant therapy, resulting in hemorrhagic complications and mortality., ${ }^{2,3}$ Aortic CT-angiography should have been made for correct diagnosis. In our case, operative examination confirmed the intramural hematoma
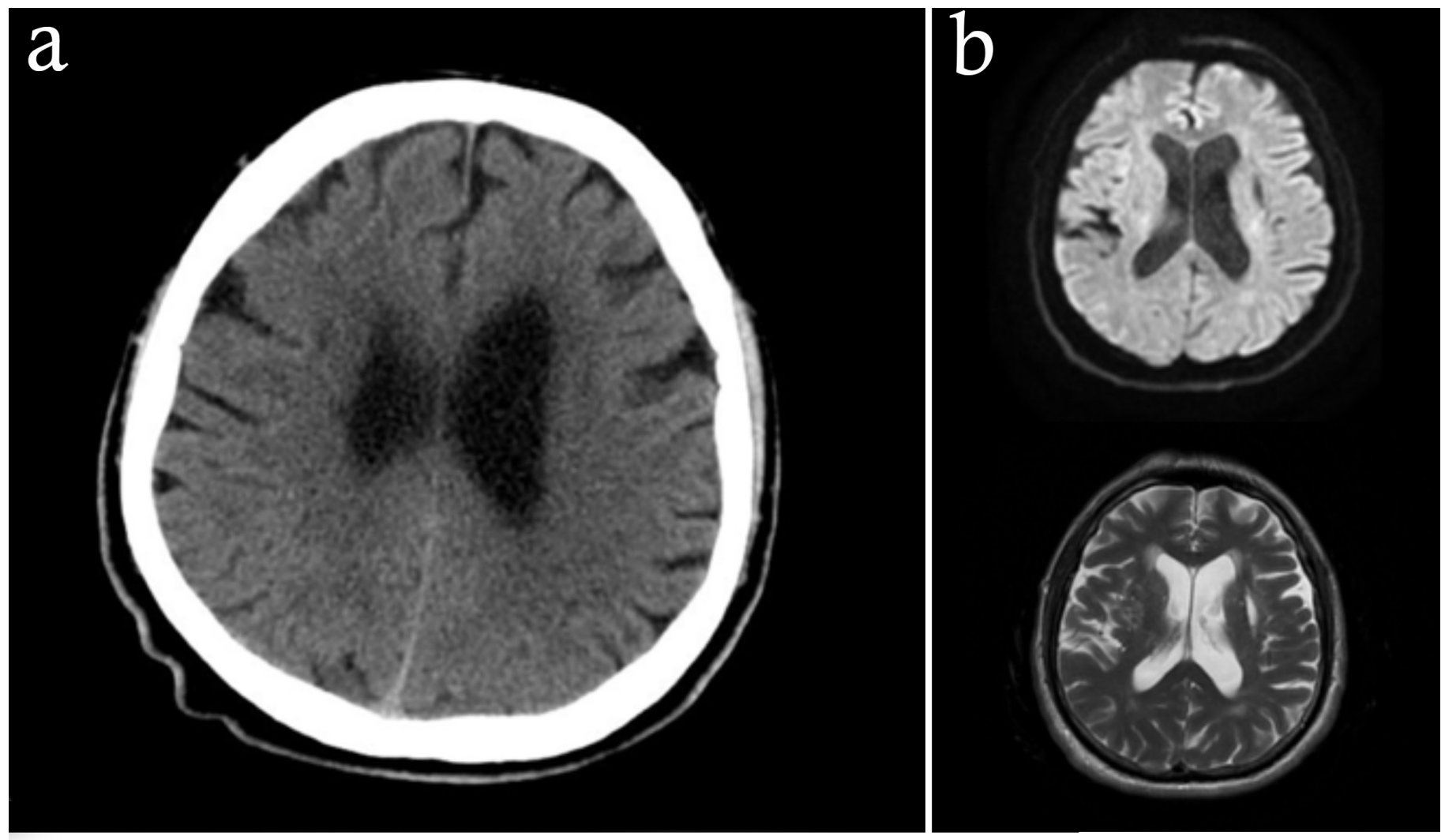

Figure 1: Head computed tomography (CT) (a) and magnetic resonance imaging (MRI-T2 and Diffusion MRI) (b) showed no remarkable findings 
of the ascending aorta, which extends retrograde from the subclavian tearing site to the descending aorta. Although we did not find an intimal flap by echocardiography, long intramural hematoma along the whole length of the lesion was suspected to be a complication of AAS. In this case, paraparesis as a result of the spinal ischemia could have occurred secondary to the compression or occlusion of the spinal artery or intercostal arteries by the hematoma. Different neurophysiological characteristics have been described according to the anatomical position of the spinal infarction: anterior spinal artery syndrome is the most clinically frequent. Its severity depends on the transversal extension of the infarction. At most the anterior two-thirds of the spinal cord are involved, with massive motor deficit below the lesion, constant vesical-sphincter disorders, and reduced sensitivity to pain and temperature below the wound. Longitudinal extension of the infarction is determined by the quality of the anastomoses of the anterior spinal artery. ${ }^{4,5}$

Paraplegia or paraparesis after AAS is a rare manifestation. If a patient complains of neurologic symptoms after AAS, clinicians should thus consider many conditions in the differential diagnosis, such as embolism of the spinal artery, acute stroke and spinal infarction due to AAS complicated with myocardial infarction. One study including 44 patients with spinal ischemia or infarction reported that AAS occurred in two patients (4.4\%), which was not extremely rare. ${ }^{6}$ When the dissection was spiral it was more probably responsible for avulsion of the intercostal arteries, which represented a factor of poor perfusion. ${ }^{7}$ Therefore, evaluation for the cause of non-traumatic paraplegia or paraparesis must be included in aortic imaging studies such as CT. Also, only an imaging study can find AAS as a cause of paraplegia or paraparesis, especially in patients with painless aortic dissection with paraplegia. ${ }^{8}$ In humans, embolic disease can occur secondary to chronic aortic dissection with resultant hypercoagulability or from thrombi formed at the site of the aneurysm. "Blue toe" syndrome in humans is the result of those showered emboli causing distal limb ischemia. ${ }^{9,10}$ Thrombi theoretically form secondary to weakening of the intimal layer of the aortic aneurysm, which allows endothelial activation of coagulation factors. ${ }^{9}$ Although aortic dissection can cause paraparesis secondary to deranged spinal blood

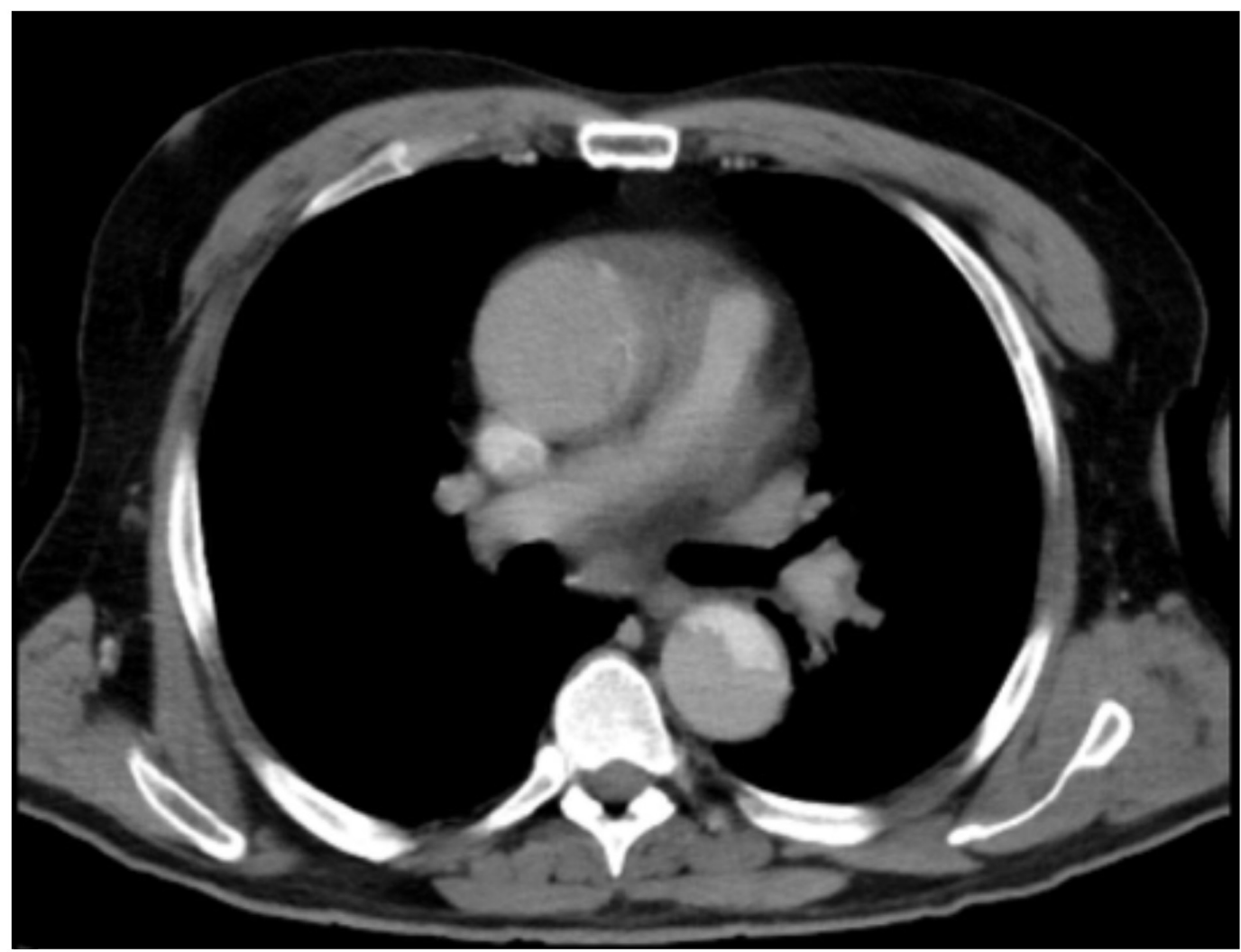

Figure 2: A dissection detected in the ascending aorta, which may be retrograde extended from the subclavian tearing site to the descending aorta complicated with neurologic complaints was observed. 
flow or femoral arterial emboli; given the existence of unilateral arterial pulse deficit and bilateral neurologic complaints, the latter was thought to be a less important factor in this case.

In conclusion, the correct diagnosis of AAS has many potential pitfalls because AAS may mimic myocardial ischemia, stroke, heart failure and shock. In this case, balancing treatment for aortic dissection versus arterial thromboembolism was a clinical challenge. Acute aortic dissection is a clear contraindication for the use of antithrombotic and thrombolytic, such as heparin or tPA, given the inhibition of clotting mechanisms that potentially could stem further dissection. Overall prognosis for aortic dissection is assumed to be poor without surgical intervention. This case also highlights the importance of serial physical examination. Careful interpretation of the imaging results and a high index of suspicion in patients with neurologic complaints are therefore critical. To make the right decision about the treatment of AAS with neurologic complications, the benefits and risks should therefore be considered in individual patients.

\section{Conflict of Interests}

Authors declare that there are none.

\section{References}

1. Hagan PG, Nienaber CA, Isselbacher EM, Bruckman D, Karavite DJ, Russman PL, Evangelista A, Fattori R, Suzuki T, Oh JK, Moore AG, Malouf JF, Pape
LA, Gaca C, Sechtem U, Lenferink S, Deutsch HJ, Diedrichs H, Marcos y Robles J, Llovet A, Gilon D, Das SK, Armstrong WF, Deeb GM, Eagle KA. The International Registry of Acute Aortic Dissection (IRAD): new insights into an old disease. JAMA. 2000 Feb 16;283(7):897-903. doi: 10.1001/jama.283.7.897.

2. Butler J, Davies AH, Westaby S. Streptokinase in acute aortic dissection. BMJ. 1990 Feb 24;300(6723):517-9. doi: 10.1136/bmj.300.6723.517.

3. Melchior T, Hallam D, Johansen BE. Aortic dissection in the thrombolytic era: early recognition and optimal management is a prerequisite for increased survival. Int J Cardiol. 1993 Nov;42(1):1-6. doi: 10.1016/01675273(93)90095-X.

4. Fried LC, Aparicio O. Experimental ischemia of the spinal cord. Histologic studies after anterior spinal artery occlusion. Neurology. 1973 Mar;23(3):289-93. doi: 10.1212/wn1.23.3.289.

5. Garcin R, Godlewski S, Lapresle J. [Acute pseudopoliomyelitic myelomalacia. (Massive necrotic lesions of the anterior horns in the lumbosacral spinal cord)]. Rev Neurol (Paris). 1969 Oct;121(4):487-91.

6. Cheshire WP, Santos CC, Massey EW, Howard JF Jr. Spinal cord infarction: etiology and outcome. Neurology. 1996 Aug;47(2):321-30. doi: 10.1212/ wn1.47.2.321.

7. Waldrop JE, Stoneham AE, Tidwell AS, Jakowski RM, Rozanski EA, Rush JE. Aortic dissection associated with aortic aneurysms and posterior paresis in a dog. $\mathrm{J}$ Vet Intern Med. 2003 Mar-Apr;17(2):223-9.

8. Inamasu J, Hori S, Yokoyama M, Funabiki T, Aoki K, Aikawa N. Paraplegia caused by painless acute aortic dissection. Spinal Cord. 2000 Nov;38(11):702-4. doi: 10.1038/sj.sc. 3101087 .

9. Grenvick A, Ayres SM, Holbrook PR, Shoemaker WC, eds. Textbook of Critical Care. 4th ed. Philadelphia: WB Saunders; 2000:1118-9, 1747-52.

10. Karmody AM, Powers SR, Monaco VJ, Leather RP. "Blue toe" syndrome. An indication for limb salvage surgery. Arch Surg. 1976 Nov;111(11):1263-8. doi: 10.1001/archsurg.1976.01360290097015. 